\title{
Evaluation of models for tube material characterization with the tube bulging test in an industrial setting
}

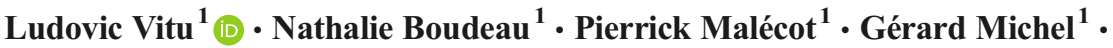 \\ Aurélien Buteri $^{2}$
}

\begin{abstract}
It is now well recognized that the material data obtained from a tensile test is less appropriate than those from a Tube Bulging Test (TBT) for a finite element simulation of tube hydroforming. However, the manufacturers still use classical data (often tensile test data) for designing metal operations due to the lack of standard for the TBT and a more complex post processing analysis of experimental measures. Getting the hardening curve from the tube bulging test requires the use of an analytical or numerical model. In this paper, three models for post-processing measures obtained from the TBT are compared based on the same experimental procedure. Thanks to a preliminary step, consisting of the validation of the analytical models through the use of finite element simulations of the TBT, it highlights that the results obtained for the local (stress and strain) and global components (the thickness distribution along the tube and the deformed tube profile) are very close, whatever the models. The test configuration (die radius and free length) seems to have no significant impact on the resulting stress-strain curve for the three models. The three models are used for post processing tube bulging tests performed on AISI304, INCONEL and Copper tubes validating their capacity for tube characterization on different materials. Finally, this study demonstrates that the Boudeau-Malécot Model can be used to obtain hardening curve from TBT without a loss of accuracy compared to
\end{abstract}

Nathalie Boudeau

nathalie.boudeau@ens2m.fr

1 FEMTO-ST Institute, Department of Applied Mechanics, UMR 6174, ENSMM, CNRS, Université Bourgogne Franche Comté, 24 chemin de l'Epitaphe, F 25000 Besançon, France

2 APERAM, Research Center, F 62230 Isbergues, France more complex post-processing models and with an important gain of quality compared to tensile test.

Keywords Tube bulging test $\cdot$ Hydroforming $\cdot$ Hardening curve $\cdot$ Analytical model $\cdot$ Finite element simulation

\section{Introduction}

Tube hydroforming process consists of forming a tube inside a closed and shaped cavity by applying an internal pressure. The internal pressure is most of the time combined to a compression axial force, to produce complex shapes and to minimize the critical thinning. This technology is of particular interest for industry because it allows the achievement of complex hollow shaped parts with a reduced number of welding spots and a higher structural quality. Automotive industry is one of the main users of this technology with different applications such as exhaust system, chassis, body and safety parts (made of stainless steels, low to medium carbon steels or aluminium alloys) shaped with pressure [1].

By contrast, its industrial implementation is relatively complex mainly due to the expensive price of the equipment and the large number of parameters, which must be set. In particular, the axial compressive force - internal pressure path must be optimised to avoid bursting, wrinkling and buckling by using the Process Window Diagram [2]. Finite element simulations are widely used for determining the process variables and optimizing the final component quality; for that more and more complex algorithms are coupled with FE code such as random search techniques [3]. But numerical results are partly affected by the modelling of material behaviour [4]. As materials harden differently with their loading history [5], material data obtained from classical tensile test are unsatisfying for performing reliable tube hydroforming simulations. That is 
why the tube bulging test has been developed, where a tube is freely expanded by applying an internal pressure (Fig. 1).

To get material data such as the stress-strain curve from this advanced experimental test, researchers have developed advanced techniques. The technical fashion for strain measurements is the Digital Image Correlation (DIC) [6]. Even if the Measurement Departments in the industry increasingly use this kind of systems, their cost is not negligible and the closed dies used in TBT are not adapted for measures with DIC systems. The first tests we carried out gave surprising results like a negative meridian strain during tube bulging leading to an underestimation of the equivalent true strain (Fig. 2). A recent paper [8] presents specific developments for performing stereo-correlation on 3D complex surfaces; it proves that classical systems are not entirely satisfying for DIC measurements on tube under pressure. Moreover, in the TBT developed in our laboratory, the observation window is relatively small that introduces an additional difficulty. For stress evaluation, the up-to-date approach is the reverse identification method based on the minimization of the error between experimental measures and finite element (FE) simulations results based on a FE model of the TBT [9]. This technique is efficient but the computation time is quite long.

A more conventional and faster method is the use of analytical or numerical models. Many of models can be found in the literature. A short overview is given in the following paragraphs.

One of the first paper on the experimental application of tube formability and tube material characterization with the TBT is credited to Altan's research group in 2000 [10]. The TBT is carried out on a tube loaded with an internal pressure and clamped at its two extremities in such a way as only the centred area of the tube is bulged. Tests are conducted to determine the minimal value of pressure leading to plastic deformation, and the maximal one corresponding to bursting. Between these two values, different intermediate pressures are tested in order to estimate the equivalent strain and calculate the equivalent stress from Fuchizawa's eqs. [11], according

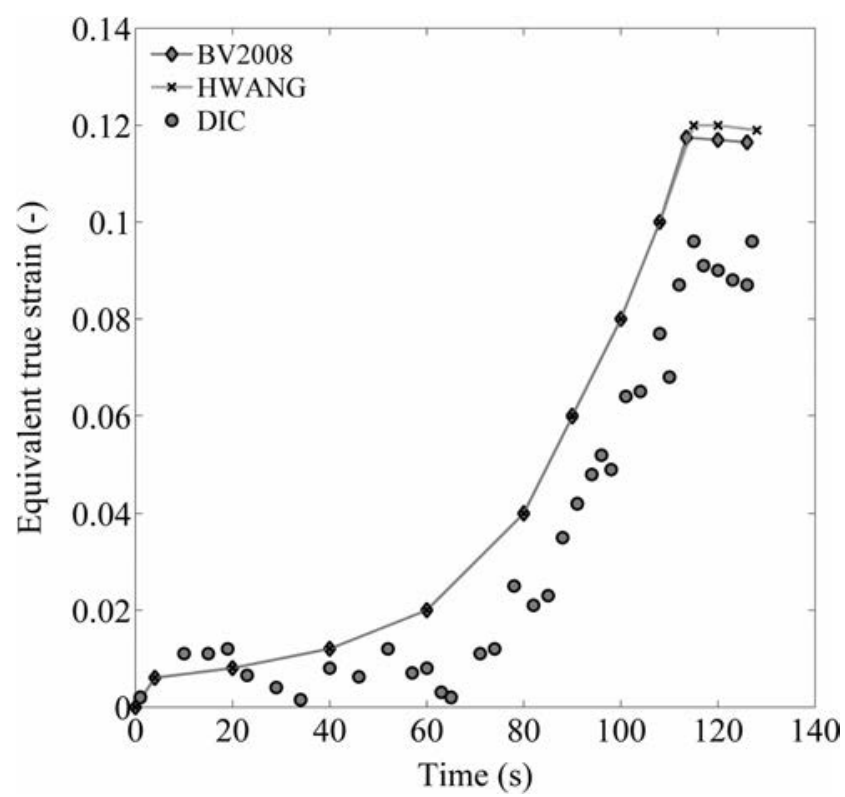

Fig. 2 Comparison of the evolution of equivalent true strain during tube bulging test obtained from Hwang 2007, Boudeau-Velasco 2008 models and DIC (Aramis) (from [7])

to the current thickness and the current bulged height at the pole. These experimental data combined to finite element simulation permits the determination of the material parameters of the Swift's hardening law with the help of a least square method. More recently, Bortot et al. [12] used a similar experimental procedure with a more advanced analytical model for conducting the material characterization. The main criticism about these approaches is that they need of numerous specimens to get the hardening curve.

By consulting the literature, it has been found that different bulging apparatus have been developed with different boundary conditions. The tube can be fixed [10, 13-15], free [14], closed [11], free and closed [11], loaded with an axial compression force $[15,16]$ or not. In a recent paper from He et al. [17], it is shown that the obtained stress states are equivalent for closed-end and fixed-end configurations.

The experimental procedure can also be different from a laboratory to another. The experiments are more or less
Fig. 1 Schematic view of the tube bulge test principle

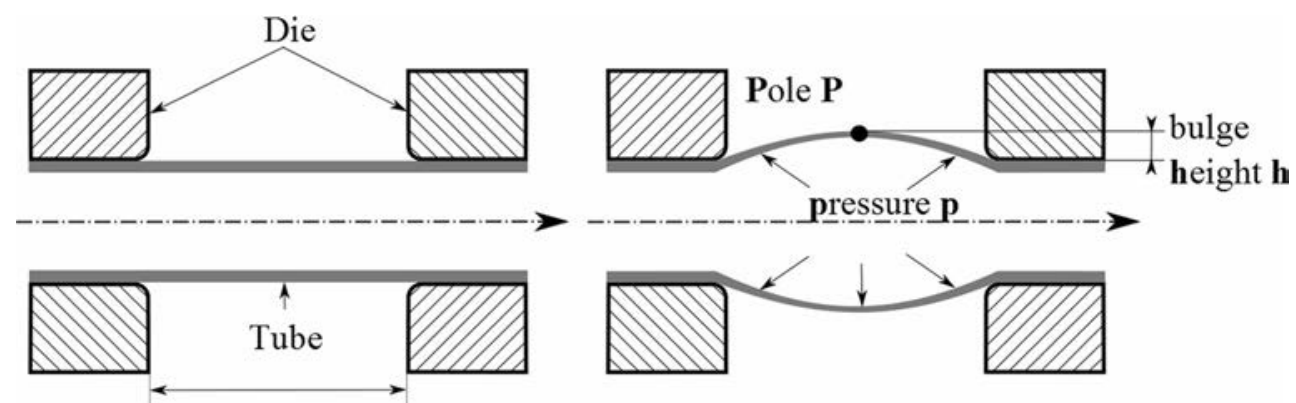

free bulging Length $\mathbf{L}$ 
complex and time/material consuming. In all the bulge tests, the pressure is measured during the experiment. On the other hand, the evaluation of strains needs the knowledge of the geometry of the deformed tube. In [11], the meridian radii is measured with three displacement transducers placed along the tube, centred to the pole of the bulged tube, as a spherometer and two strain gauges are used in [18]. A unique displacement sensor is necessary in $[15,19]$ for measuring the bulge height at the pole. In [12], tubes are bulged at different pressure levels and cut to carry out different measures on the deformed samples.

Strain evaluation is often based on the assumption of plastic incompressibility; it is then necessary to measure or evaluate the tube thickness at the pole represented by the point $\mathrm{P}$ in Fig. 1. The pole thickness can be measured continuously during the test [11], with the help of a thickness sensor. But it can also be measured on tubes after testing different pressures [10, 13]. Based on experimental observations, thickness distribution can be defined by a quadratic function based on thicknesses of two characteristic points (the pole and the boundary between the die radius and the free expanding area of the tube) such as in [13]. It can also be evaluated from the constancy of the material volume [15].

According to the approach, the geometrical representation of the bulged tube can differ from one to another. Nevertheless, for the majority, the profile of the deformed tube is assimilated to an arc of circumference $[10,15,16,18]$ or an elliptical profile [13].

Finally, stress analysis is usually based on the mechanical equilibrium of an elementary volume centred at the pole $[10$, $16,18,20]$, except in [15] in which the obtained stress field is not limited to the pole region of the deformed tube.

At present, no actual guidelines exist for describing the experimental route and giving recommendations on the method for processing the experimental results. This lack of rigorous procedure can be an obstacle for an industrial use of the tube bulging test for tube material characterization.

In this paper, three models are studied: Hwang 2007 [13], Boudeau-Velasco 2008 [15] and Boudeau-Malécot 2012 [21]. All three are theoretical models for getting the stress-strain curve from the experimental pressure - bulge height curve without the help of finite element simulations. They can easily be used in an industrial laboratory for a rapid tube characterization.
In one hand, the aim of the paper is to compare the three selected models of the literature, whose main differences and equivalences are summarised in Table 1. These models are based on the same experimental procedure allowing mutual comparison: two fixed extremities and only a central deformation of the tube induced by internal pressure. In any case, the pressure and the bulging height are continuously measured, in order to obtain the strain-stress curve thanks to only one test. Models [13] and [15], unlike model [21], are both semi-analytical models and no fully analytic since a non-linear equation has to be solved. Model [21] is a full analytical model and experimental results can be proceeded with a spreadsheet application (Excel@-type) that can present an advantage in an industrial context. From numerical simulations, the TBT configuration and the capability of the three selected models to characterize the tube material are studied, where the die radius and the free bulging length define the test configuration.

In the other hand, its application to different tube materials is proposed.

As a first step, the study is focused on isotropic material, as the tube specimen has undergone annealing heat treatment. In the last section, it is shown that the studied models can also be used for anisotropic materials.

The paper is organized as follows. Second section presents the theoretical basis of the three selected models. Then, third section broaches the procedure used to validate and compare the three models on different test configurations. This procedure is mainly based on finite element simulations and the fourth section is devoted to the presentation of the results and their discussions. In fifth section, the three selected models are run for post-processing experimental results of the tube bulging test performed on Copper, Stainless Steel and Inconel tubes and the consideration of anisotropy is discussed.

\section{Theory}

Three models have been selected for these comparisons: Hwang's model [13, 22], Velasco \& Boudeau's model [15] and its simplified form [21].

Table 1 Summary of the differences and equivalences of the three models

\begin{tabular}{|c|c|c|c|}
\hline Models & Geometrical representation & Strain evaluation & Stress evaluation \\
\hline Hwang-Lin 2007 & \multirow{3}{*}{$\begin{array}{l}\text { Elliptical profile }+ \text { Thickness repartition law } \\
\text { Circular profile }\end{array}$} & \multirow{3}{*}{$\begin{array}{l}\text { Volume constancy }=\text { Non-linear } \\
\text { set of equations } \\
\text { Meridian strain constant }\end{array}$} & Fuchizawa equations defined at the pole \\
\hline Velasco-Boudeau 2008 & & & Equilibrium of elementary volumes \\
\hline Boudeau-Malécot 2012 & & & Valuable all over the tube \\
\hline
\end{tabular}




\section{Hwang's model}

Hwang's model is based on three particularities:

- The meridian profile of the bulged tube is represented by an arc of ellipse in its free expanding zone and by the die radius at the end of the guidance zone. The ellipse is defined by its major axis $R_{Z}$ and its minor axis $R_{P}$ :

$$
\left(\frac{r}{R_{p}}\right)^{2}+\left(\frac{z}{R_{z}}\right)^{2}=1
$$

- The die radius $R_{d}$ and a point $E\left(R_{E}, Z_{E}\right)$ are considered; the point $\mathrm{E}$ corresponds to the boundary between the free bulging zone and the area under the die radius (Fig. 3a).

- Thickness along the meridian profile of the tube follows a quadratic law defined through the pole thickness at point $\mathrm{P}, t_{P}$ and the thickness at point $E, t_{E}$ :

$$
\left\{\begin{array}{l}
t(z)=t_{P}+\left(\frac{\omega}{\omega_{E}}\right)^{2}\left(t_{E}-t_{P}\right) \\
\omega=\tan ^{-1}\left[\frac{R_{Z}}{R_{P}} \cdot \tan \gamma\right] \\
\omega_{E}=\tan ^{-1}\left(\frac{Z_{E}}{R_{E}}\right)
\end{array}\right.
$$

where $\gamma$ is the elliptical parameter.

Coordinates of point $E$ can be determined numerically from Eq. 1, and $t_{P}$ and $t_{E}$ can be numerically evaluated using the plastic incompressibility or conservation of volume as described in [12]:

$V_{\text {free }}^{i+1}+\Delta V_{\text {contact }}=V_{\text {free }}^{i}$

where $V_{\text {free }}^{i}$ and $V_{\text {free }}^{i+1}$ correspond to the volume of material in the free region at times $i$ and $i+1$ respectively, and $\Delta V_{\text {contact }}$ the variation of volume of material in contact with the die radius between these two times (Fig. 3b).

The volume of material in the free region is given by:

$$
\left\{\begin{array}{l}
\mathrm{V}_{\text {free }}=2 \pi \int_{0}^{\gamma_{E}} R_{P} \cdot \cos \gamma \cdot t(z) \cdot \sqrt{\left[R_{P} \cdot \sin \gamma\right]^{2}+\left[R_{Z} \cdot \cos \gamma\right]^{2}} d \gamma \\
\gamma_{E}=\tan ^{-1}\left[\frac{R_{P} \cdot Z_{E}}{R_{Z} \cdot R_{E}}\right]
\end{array}\right.
$$

The increment of volume of tube in contact with the die is illustrated in Fig. $3 \mathrm{~b}$ and can be expressed as follows:

$$
\begin{gathered}
\Delta \mathrm{V}_{\text {contact }}=2 \pi t_{E}(i)\left[R_{0}+R_{d}\left(1-\cos \phi_{E}(i)\right)-0.5 t_{E}(i) \cos \phi_{E}(i)\right] \\
{ }^{*}\left[R_{d}+0.5 t_{E}(i)\right] \Delta \phi_{E}
\end{gathered}
$$

where $R_{0}$ stands for the initial radius and $t_{E}$ for the initial thickness of the tube at point $\mathrm{E}$, and where $R_{d}$ corresponds to the die radius. $\phi_{E}$ represents the angle defined in Fig. $3 \mathrm{a}$.

So it is possible to evaluate the strains at the pole $P$ :

$\left\{\begin{aligned} \varepsilon_{\theta} & =\ln \left(\frac{R_{P}-0.5 t_{P}}{r_{0}-0.5 t_{0}}\right) \\ \varepsilon_{r} & =\ln \left(\frac{t_{P}}{t_{0}}\right) \\ \varepsilon_{z} & =-\left(\varepsilon_{\theta}+\varepsilon_{r}\right)\end{aligned}\right.$

Stresses at the pole, for an internal pressure $p$, are calculated from the following equations:

$\left\{\begin{aligned} \sigma_{z} & =\frac{p\left(\rho_{\theta}-t_{P}\right)^{2}}{2 t_{P}\left(\rho_{\theta}-0.5 t_{P}\right)} \\ \sigma_{\theta} & =\frac{p\left(\rho_{\theta}-t_{P}\right)}{2 t_{P}\left(\rho_{z}-0.5 t_{P}\right)}\left(2 \rho_{z}-\rho_{\theta}-t_{P}\right)\end{aligned}\right.$

with:

$\left\{\begin{array}{l}\rho_{z}=\frac{R_{Z}^{2}}{R_{P}} \\ \rho_{\theta}=R_{P}\end{array}\right.$

More details can be found in [20, 22].

\section{Boudeau \& Velasco's model}

This model considers that the free bulging zone of the tube deforms as two arcs of circumference: one of radius $R$ and centre $C(y, 0)$ in the meridian direction; another of radius $r$ and centre $O(0,0)$ in the circumferential direction (Fig. 4). It assumed that the external (denoted by ${ }_{e}$ index) and internal (denoted by ${ }_{. i}$ index) surfaces of the tube deform in such a way:

$\left\{\begin{array}{l}R_{q}=\frac{d^{2}+h_{q}^{2}}{2 \cdot h_{q}} \\ y_{q}=\frac{d^{2}-h_{q}^{2}-2 h_{q} r_{q}}{2 h_{q}}\end{array} \quad\right.$ with $q=e, i$ 
Fig. 3 a Hwang's model, b Calculation of the conservation of volume (from [13])
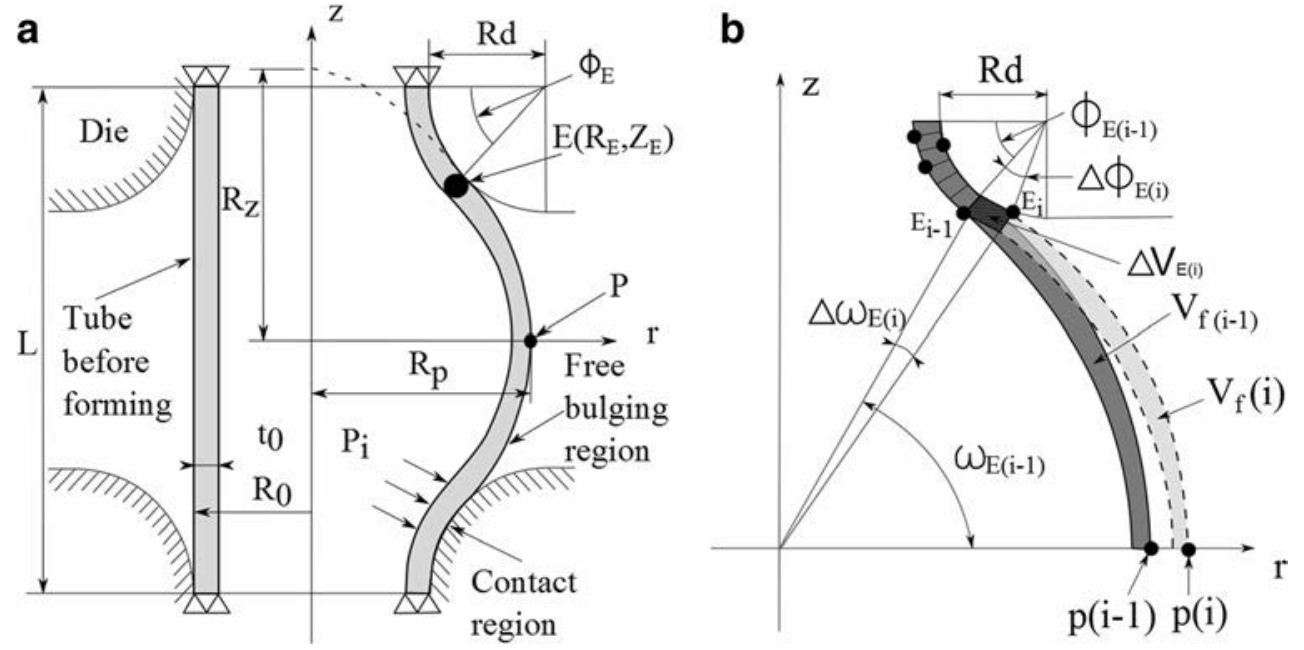

with:

and the plastic incompressibility condition gives finally:

$\left\{\begin{array}{l}r_{e}=r_{0}+h_{e} \\ r_{i}=r_{0}-t_{0}+h_{i}\end{array}\right.$

where $r_{0}$ and $t_{0}$ are the initial radius and thickness of the tube, $d$ the half-length of the window in the die for free bulging, $h_{e}$ the bulge height measured on the external surface of the tube and $h_{i}$ the internal bulge height.

So at each point of the tube, the difference between the internal and external surface gives the current thickness. At the pole it gives:

$t_{P}=t_{0}+h_{e}-h_{i}$

The volume of material that deforms is then:

$\left\{\begin{array}{l}V_{\text {material }}=V_{e}-V_{i} \\ V_{q}=2 \pi \int_{0}^{d} Y_{q}(Z) d Z \\ Y_{q}(Z)=-y_{q}-\sqrt{R_{q}^{2}-Z^{2}}\end{array}\right.$

$V_{\text {material }}\left(d, r_{i}, r_{e}, y_{i}, y_{e}, h_{i}, h_{e}\right)=V_{0}=\pi d\left(r_{e}^{2}-r_{i}^{2}\right)$

Equation 13 leads to a non-linear equation where the unique unknown is $h_{i}$; a Newton-Raphson algorithm permits its evaluation. Once $h_{i}$ is determined, the internal and external meridian profiles are known and the thickness can be evaluated in the whole tube. The following relations evaluate the strains:

$$
\left\{\begin{array}{l}
\varepsilon_{r}=\ln \left(\frac{t(Z)}{t_{0}}\right) \\
\varepsilon_{\theta}=\ln \left(\frac{Y(Z)}{r_{0}}\right) \\
\varepsilon_{z}=-\left(\varepsilon_{\theta}+\varepsilon_{r}\right)
\end{array}\right.
$$

Fig. 4 Setting of the Velasco \& Boudeau's model (from [15])

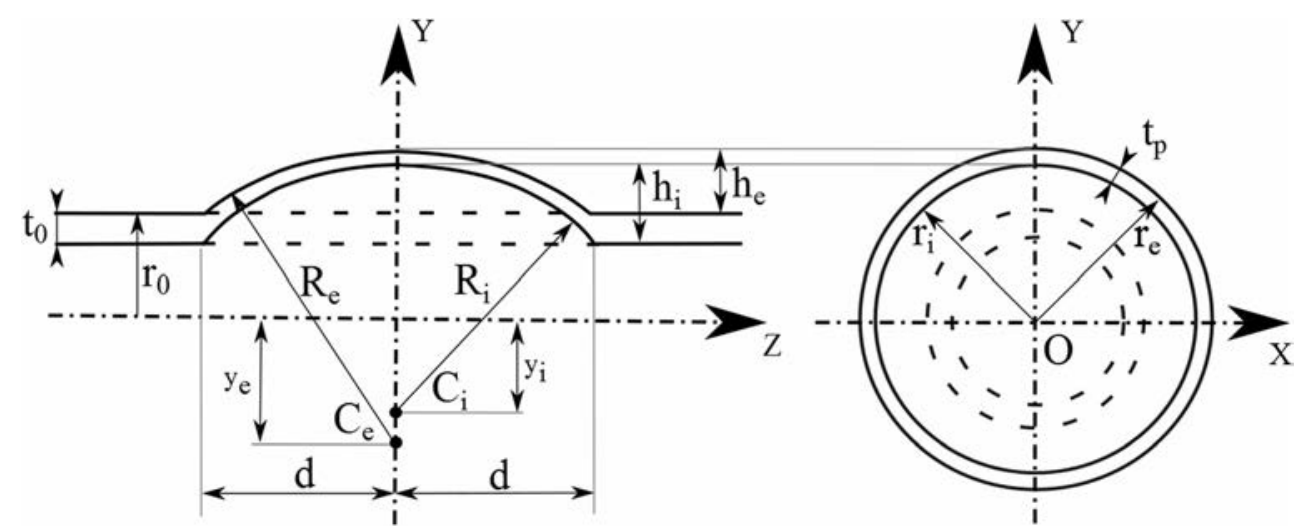


Stress state is determined by writing the mechanical equilibrium of several elementary volumes detailed in [15] leading to the following expressions:

$$
\left\{\begin{array}{l}
\sigma_{z}=\frac{p Y}{2 t \cos \phi} \\
\sigma_{\theta}=\frac{p Y}{t \cos \phi}\left[1-\frac{Y}{2 R \cos \phi}\right]
\end{array}\right.
$$

where $\phi \in\left[0 ; \phi_{\max }\right]$ and $\phi_{\max }=\sin ^{-1}\left(\frac{d}{R}\right)$.

\section{Boudeau \& Malécot's model}

This model [21] is an evolution of Velasco \& Boudeau's model, which is a semi-analytic model, as we need a Newton-Raphson algorithm to solve the non-linear equation Eq. 13. The goal of this evolution is to obtain a full analytical model in the end. This is achieved by suppressing the non-linearity. For that, a mean value for $\varepsilon_{z}$ is considered; numerical results have revealed a very weak evolution of the meridian strain $\varepsilon_{z}$ along the tube and it was decided to calculate $\varepsilon_{z}$ by considering the global variation of the free tube length. The expressions for the strains are now:

$\left\{\begin{array}{l}\varepsilon_{z} \approx \ln \left(\frac{R \cdot \phi_{\max }}{d}\right)=c s t e \\ \varepsilon_{\theta}=\ln \left(\frac{Y(z)}{r_{0}}\right) \\ \varepsilon_{r}=-\left(\varepsilon_{z}+\varepsilon_{\theta}\right)\end{array}\right.$

Thus, the distribution of thickness in the tube is obtained from the radial strain $\varepsilon_{r}$ :

$t(z)=t_{0} e^{\varepsilon_{r}(z)}$

Stresses can be calculated in the same way than in [15] or as following:

$$
\left\{\begin{array}{l}
\sigma_{z}=\frac{A+0.5 p Y^{2}}{t Y \cos \phi} \\
\sigma_{\theta}=Y \cdot\left[\frac{p}{t}-\frac{\sigma_{z}}{R} \cdot \cos \phi\right]
\end{array}\right.
$$

with:

$$
\left\{\begin{array}{l}
A=0, \text { if } \phi_{\max }<\phi_{\max }^{l i m} \\
A=t(0)\left(r_{0}+h\right) \sigma_{z}(0)-0.5 p\left(r_{0}+h\right)^{2}, \text { if } \phi_{\max } \geq \phi_{\max }^{l i m} \\
\phi_{\max }^{l i m}=\tan ^{-1}\left(\frac{2 d}{r_{0}-h}\right)
\end{array}\right.
$$

More details can be found in [21].

\section{Method for comparison and validation}

\section{Procedure}

In contrast to the tensile test, there are no recommendations or normalisations for performing experimental tube bulging test and post-processing its resulting experimental measures. In order to control the influence of the geometrical characteristics of the tube bulging test on the tube material characterization on one hand, and to evaluate the three selected models for post-processing the experimental results in the other, it is proposed to base the analyses on results obtained with finite element simulations.

Running finite element simulations needs data such as the loading curve (pressure vs. time) and the hardening curve of the material. For this study the reference data are based on a previous study [21] performed on a $316 \mathrm{~L}$. The tube bulging test was realized at the laboratory in its reference configuration represented by a free bulging length of $50 \mathrm{~mm}$ and a die radius of $5 \mathrm{~mm}$. The experimental loading curve was a ramp of $0.1 \mathrm{MPa} / \mathrm{s}$.

In the following, this hardening curve is called the imposed hardening curve.

For validation and comparison of the three models, a finite element simulation of the tube bulge test in its reference configuration (free bulging length of $50 \mathrm{~mm}$ and a die radius of $5 \mathrm{~mm}$ ) is performed. The data for the simulation are the loading curve and the hardening curve of the material. The result is the pressure - bulge height curve that is post-processed with the three selected models to obtain the hardening curve. Figures 5 and 6 describe the procedure:

1. The resulting stress-strain curves are compared to the imposed hardening curve used for the simulation (Fig. 5).

2. Global comparisons with experimental measures, such as the resulting tube profile and the thickness distribution along the deformed tube, complete the analysis. (Fig. 6).

3. Strain and stress fields from analytical models and FE simulations are analysed and compared (Fig. 6).

Then, we run different finite element simulations, for different test configurations, for studying the influence of the geometrical characteristics of the tube bulging test. The input data for the simulations are the same loading curve and the same hardening curve than before. Results are once again the pressure-bulging height data, which are treated with the three models. The resulting stress-strain curves are compared to the imposed hardening curve. Figure 7 explains the procedure. 


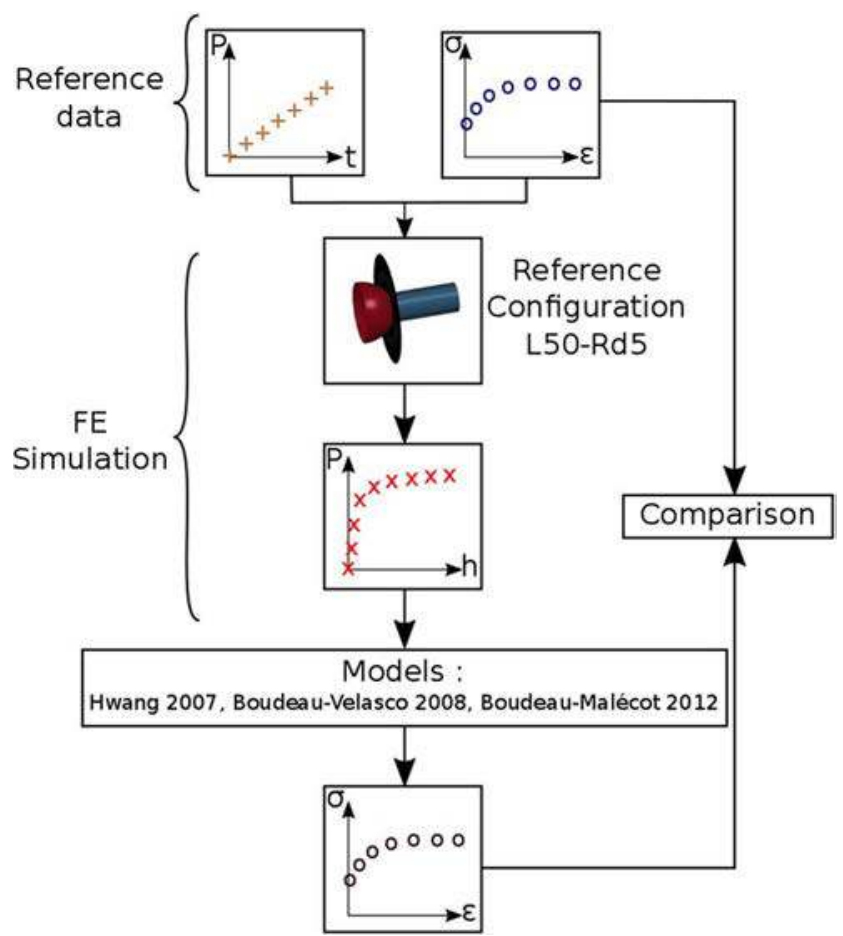

Fig. 5 Procedure for validations and comparisons of the three selected models relatively to their capability of getting the hardening curve of the tube material from the tube bulging test

\section{Finite element simulations}

Finite element simulations are performed with the LSDyna $\odot$ program based on a mesh of the full geometry of the TBT, with Belytchko-Tsay shell elements, with three integration points in the thickness. A pressureloading equivalent to $0.1 \mathrm{MPa} / \mathrm{s}$, based on the experimental conditions of the tube bulging test performed at the laboratory, is imposed. The material behaviour is considered as an elastic-plastic and isotropic one. For the tube, the hardening law is given point-by-point

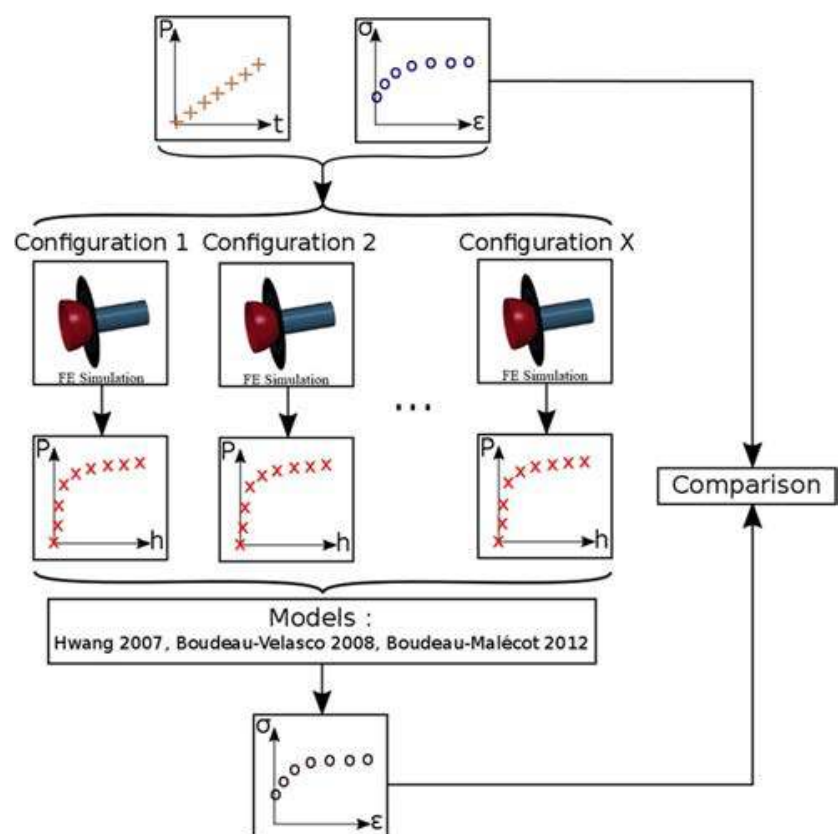

Fig. 7 Procedure for the determination of the Influence of the tube bulging test configuration on the tube material characterization

using data obtained from the literature [21]. The main information on the FE model are summarised in Table 2.

\section{Study of the influence of the test configuration}

The aim of this section is to study the influence of the geometrical characteristics (free length and die radius) used during the TBT on the obtained hardening curve. For this purpose, 5 configurations are studied, including the previously defined reference configuration $(\mathrm{L}=50 \mathrm{~mm}$ and $\mathrm{Rd} .=5 \mathrm{~mm})$. Table 3 lists the different configurations.. Finite element simulations are carried out using these different configurations and the obtained $(p, h)$ curves are post-processed with the three different models.
Fig. 6 Procedure for validations and comparisons of the three selected models relatively to their ability to represent the experimental test in terms of tube profile and thickness repartition and to predict the strain and stress fields in the tube at the pole area

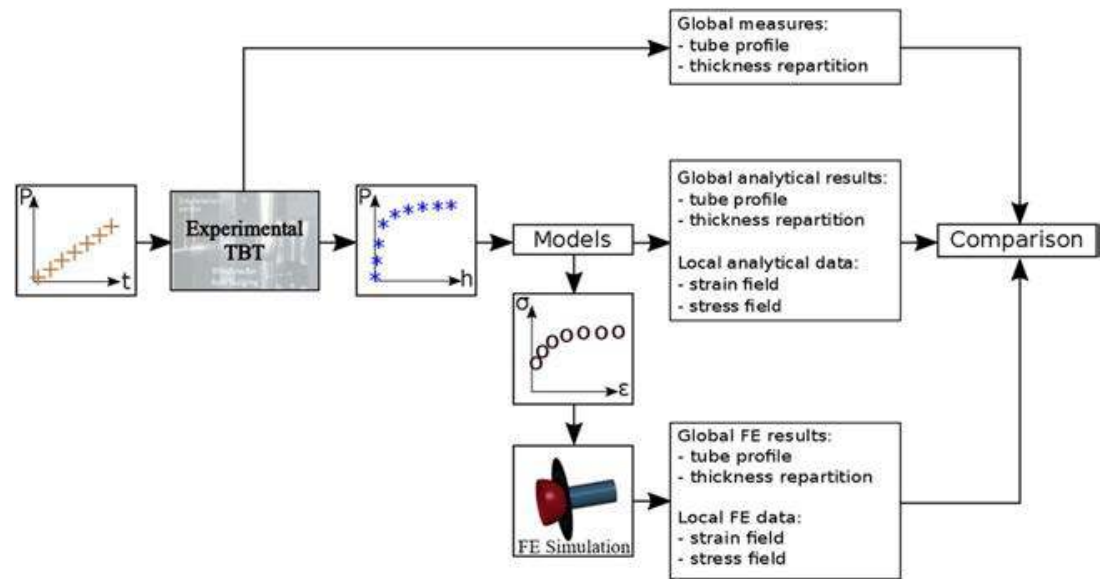


Table 2. Summary of the FE simulations with LS-Dyna $\odot$ program

$\begin{array}{ll}\text { Tube } & \text { Type: shell element } \\ & \text { Formulation: Belytchko-Tsay } \\ & \text { Number of integration points in the thickness: } 3 \\ & \text { Average size: } 1.7 \mathrm{~mm} \\ & \text { Tube Thickness: } 1 \mathrm{~mm} \\ & \text { External diameter: } 35 \mathrm{~mm} \\ & \text { Total length; } 250 \mathrm{~mm} \\ & \text { Mesh: } 8582 \text { nodes and } 8404 \text { elements } \\ & \text { Material: hardening curve described by } \\ & \text { discrete }(\sigma, \varepsilon) \text { couples } \\ & \text { Type: shell element } \\ & \text { Average size: } 1.7 \mathrm{~mm} \\ \text { Die } & \text { Mesh: } 7750 \text { nodes and } 7688 \text { elements } \\ & \text { Material: rigid } \\ & \text { Coulomb's law ( } \mu=0,1) \\ \text { Friction } & \text { Penalisation algorithm } \\ \text { Contact } & \text { Internal pressure } \\ \text { Loading } & \text { Implicit } \\ \text { Integration scheme } & \text { Ends of tube clamped. } \\ \text { Boundary conditions } & \end{array}$

\section{Results and discussion}

In this section, we adopt the following abbreviations:

- Hwang2007 for the model presented in "Hwang's model" section

- BV2008 for the model developed in "Boudeau \& Velasco's model" section

- BM2012 for the model of "Boudeau \& Malécot's model" section

\section{Validations and comparisons of models done on the reference configuration of the tube bulging test}

Different types of comparison are done in this section:

- Global comparisons are realized on data, which can easily be measured, such as the deformed tube profile and the thickness repartition along the tube (Figures 8 and 9).

Table 3 Summary of the differences configurations

\begin{tabular}{lll}
\hline $\mathrm{N}^{\circ}$ configuration & $\begin{array}{l}\text { Free bulging } \\
\text { length L }(\mathrm{mm})\end{array}$ & $\begin{array}{l}\text { Die radius Rd. } \\
(\mathrm{mm})\end{array}$ \\
\hline Reference configuration & 50 & 5 \\
1 & 50 & 7.5 \\
2 & 50 & 10 \\
3 & 40 & 5 \\
4 & 60 & 5 \\
\hline
\end{tabular}

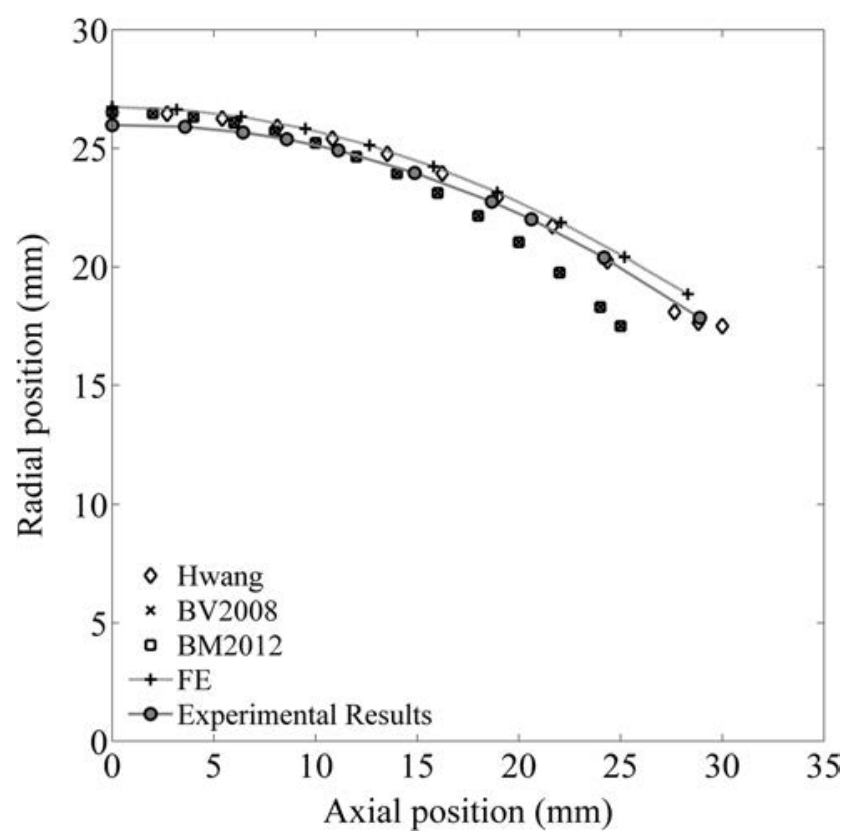

Fig. 8 Global comparisons of tube profile at the end of the bulging test

- Local comparisons are done on the components of strain and stress tensors (Figs. 10, 11, 12, 13, 14, and 15), as well as on the resulting hardening curve for the reference configuration (Figs. 18, 19, and 20).

For quantitative comparisons, the deviation of results obtained with the models is compared to the reference data. Its evaluation is based on several points distributed on a regular basis and, the maximal, minimal and mean values of the variation, expressed as a percentage, are collected in Table 4.

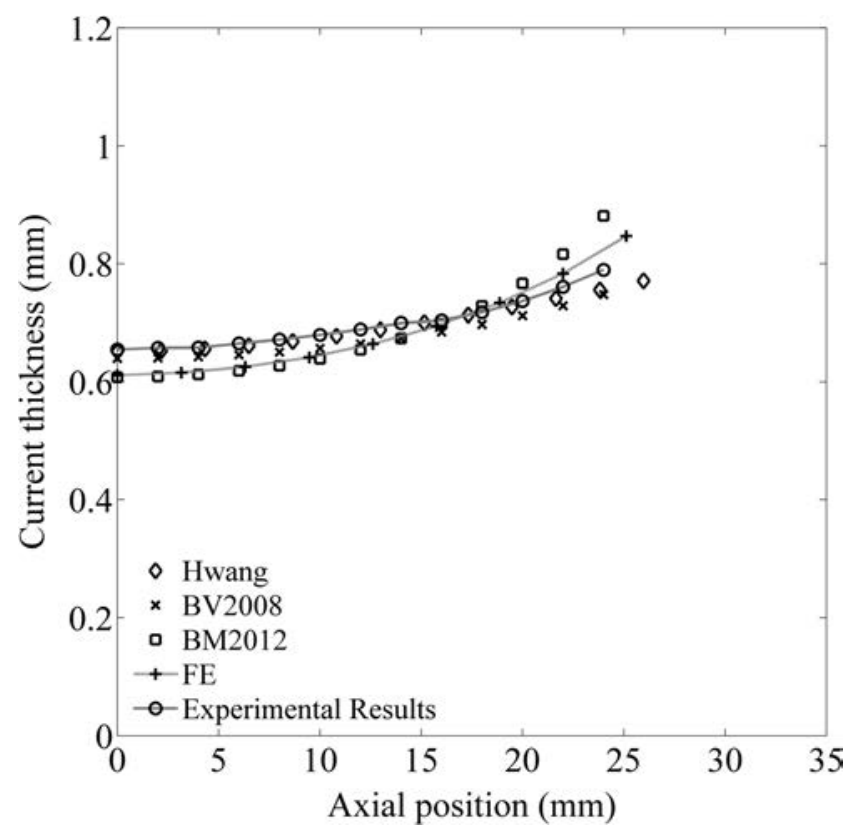

Fig. 9 Global comparisons of the thickness repartition along the bulged tube at the end of the bulging test 


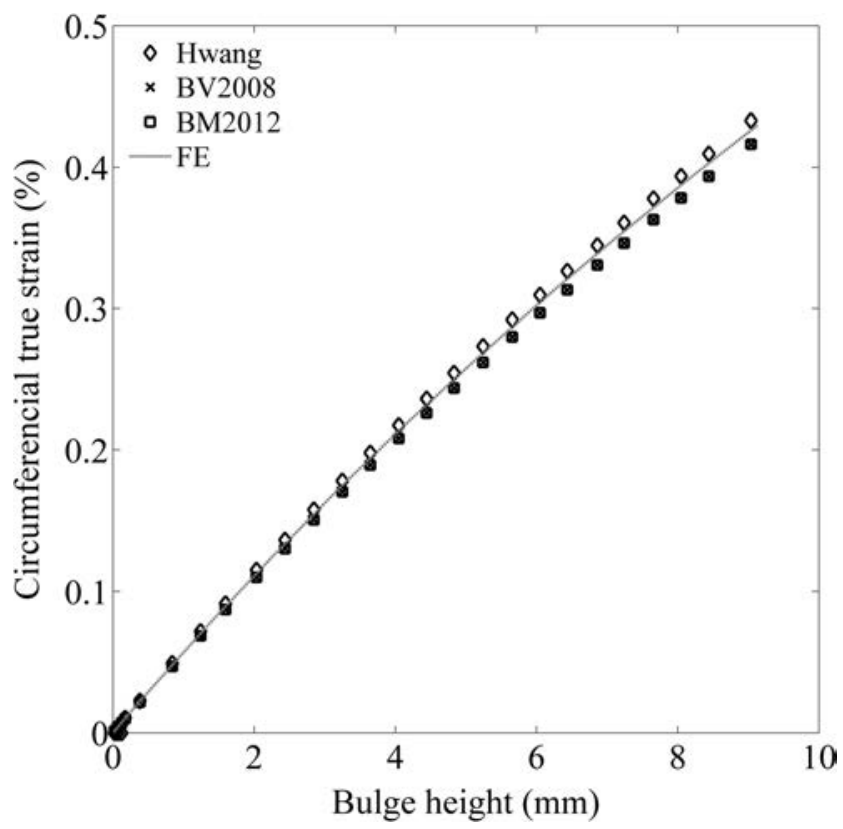

Fig. 10 Comparison of the evolution of the circumferential true strain component at the pole, with bulge height

First global comparisons are conducted. On Fig. 8, the three models almost give identical tube profiles. Indeed the comparison with FE simulation results and experimental measures is satisfying. The offset between numerical results and experimental measures is due to an overestimation of the bulge height for the maximal pressure with FE simulations. Nevertheless, Hwang's model allows representing the die radius while the other two models cannot. The value of the deviation in Table 4 (line 1) is compatible with the observation done on Fig. 8.

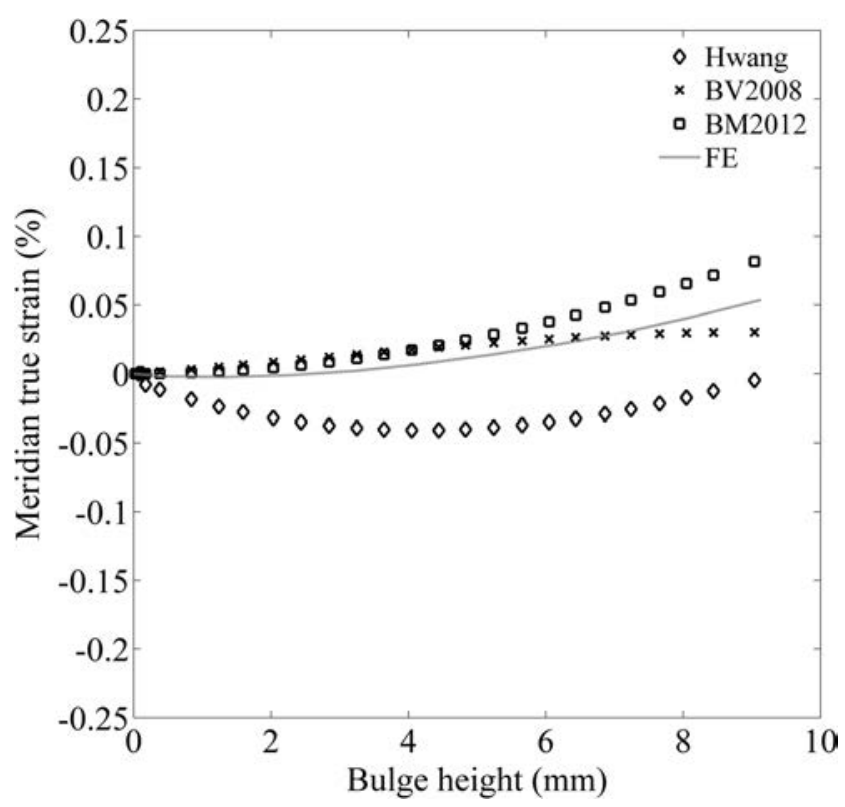

Fig. 11 Comparison of the evolution of meridian true strain component at the pole with bulge height

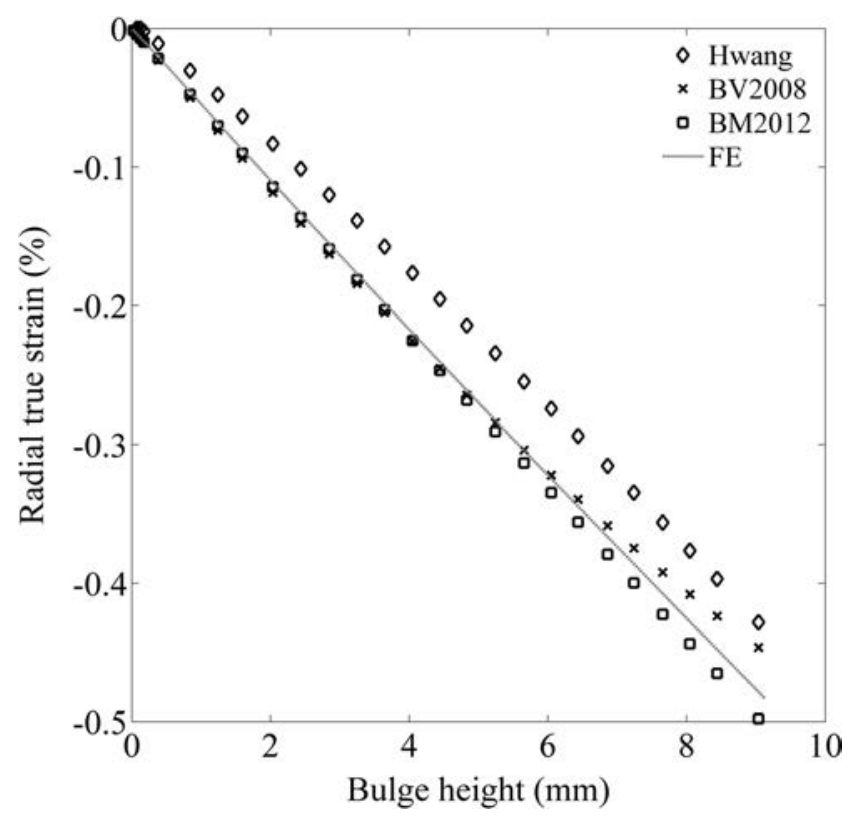

Fig. 12 Comparison of the evolution of the radial true strain component at the pole with bulge height

For the thickness repartition in Fig. 9, Hwang2007 and BV2008 are very close one to another at the pole and near the die radius, but Hwang2007 is the one, which better matches the experimental measures even if it tends to underestimate the current thickness. Quantitative data presented in line 2 of Table 4 confirm the observations done on Fig. 9. BM2012 corresponds very well to the FE results (Fig. 9), and presents a good mean value for the deviation to experimental measures

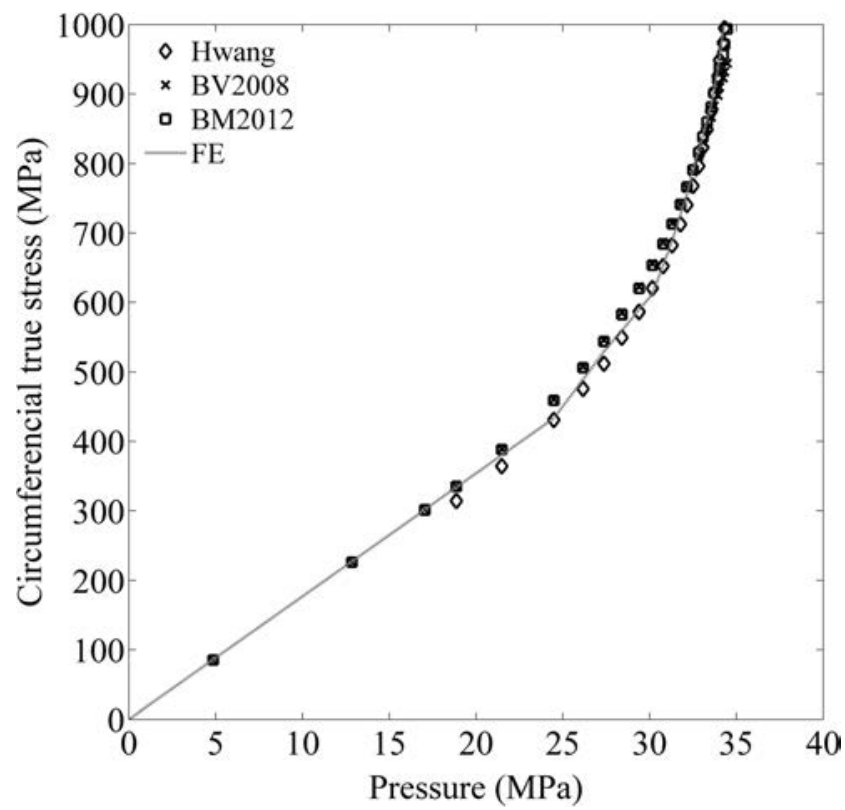

Fig. 13 Comparison of the evolution of the circumferential true stress component at the pole with pressure 


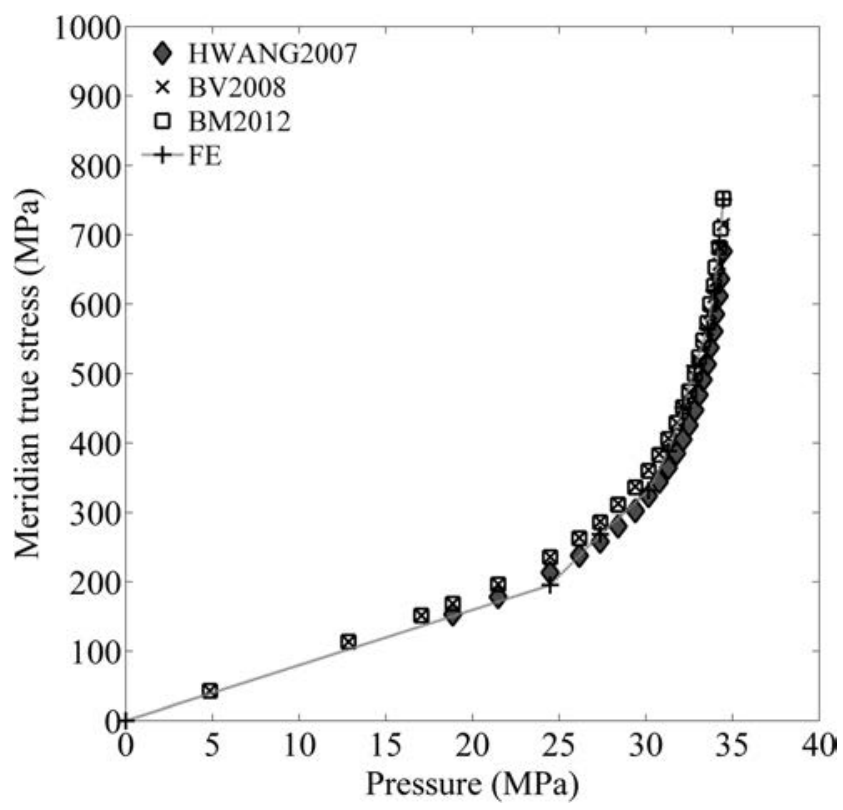

Fig. 14 Comparison of the evolution of the meridian true stress component at the pole with pressure

(line 2 in Table 4), but with an important amplitude between the minimal and the maximal deviations.

The evolution of components of strain tensor at the pole with the bulge height is compared in Figs. 10, 11, and 12. The circumferential strain evolution is very similar for the three models and very close to the FE results (Fig. 10); it is due to the method for its calculation based on Eqs. 6, 14 and 16. For the radial strain (Fig. 12), variations during the bulge test are similar whatever the model considered; Boudeau \& Velasco

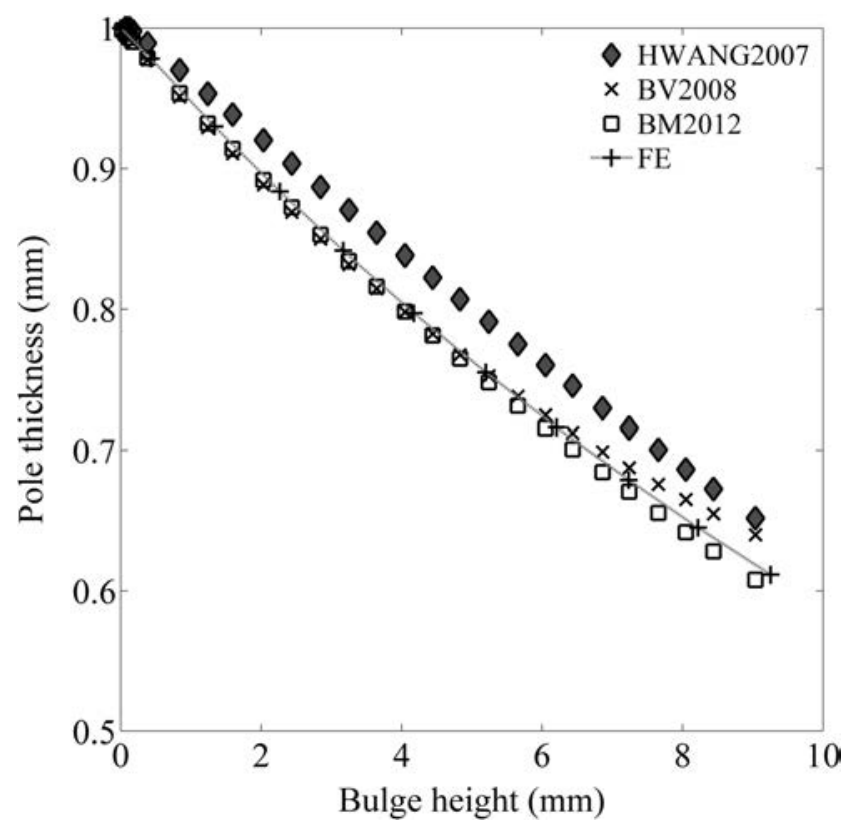

Fig. 15 Comparison of the evolution of the pole thickness with bulge height and Boudeau \& Malécot's models are close to the FE results while Hwang's model underestimates this deformation; that is in keeping with the results presented in Fig. 15 relative to the evolution of the pole thickness with the bulge height. Figure 11 illustrates the evolution of the meridian strain at the pole with the evolution of the bulge height during the test. The Boudeau-Malécot's model, which is the simplest model, gives a relatively good representation of the meridian strain's evolution. The gap between the FE results and the one obtained by post-processing the $(\mathrm{p}, \mathrm{h})$ curve with the Boudeau-Malécot's model is due to the presence of the die radius which is not taken into account in the model. The Boudeau-Velasco's model presents an evolution of the meridian strain quite different from the FE estimations; the geometrical assumptions and the difference in the radial strain evolution observed in Fig. 12 explain these variations. Surprisingly, Hwang's model gives a negative value for this strain component; the analysis of the plastic incompressibility condition enlighten this effect:

$\varepsilon_{z}=-\varepsilon_{r}-\varepsilon_{\theta}$

with $\varepsilon_{r}<0$ and $\varepsilon_{\theta}>0$. So it can be written that:

$\left\{\begin{array}{l}\varepsilon_{z}>0 \text { if }\left|\varepsilon_{r}\right|>\left|\varepsilon_{\theta}\right| \\ \varepsilon_{z}<0 \text { else }\end{array}\right.$

For the Hwang's model, we have $\left|\varepsilon_{r}\right|<\left|\varepsilon_{\theta}\right|$, due to an underestimation of thickness reduction (Fig. 15) or the radial strain (Fig. 12).

For stress components (Figs. 13 and 14), the comparison of results obtained with the three models and with the FE results are quite satisfying. Hwang2007 seems better for small bulge height as the other two models seem better for higher bulge height corresponding to the end of the test. The geometrical assumptions on the shape of the bulged tube which bases the theoretical model explains this statement; for small bulge height, an elliptical shape is closer to the reality as for an important bulge height an arc of circumference provides a better representation of the real bulged tube.

The quantitative analysis in relation with Figs. 10, 11, 12, 13, and 14 (lines 3 to 7 in Table 4) shows that:

- Results obtained with BV2008 and BM2012 are very close one to another;

- HW2007 permits a better representation of $\sigma_{z}$; for the other results, BV2008 and BM2012 also give good results with deviations less than $5 \%$ (except for $\varepsilon_{z}$ );

- The three models have difficulties to represent $\varepsilon_{z}$ (absolute main value of deviation more than 20\%). 
Table 4 Deviations between models and reference for results presented in Figs. 8, 9, 10, 11, 12, 13,14 , and 15

\begin{tabular}{|c|c|c|c|c|c|c|}
\hline $\mathrm{N}^{\circ}$ & Fig. & Reference & Models & $\operatorname{Min}(\%)$ & $\operatorname{Max}(\%)$ & Mean $(\%)$ \\
\hline \multirow[t]{3}{*}{1} & \multirow[t]{3}{*}{ Tube profile (Fig. 8) } & \multirow[t]{3}{*}{ Measure } & Hwang & -0.65 & +2.1 & +1.3 \\
\hline & & & BV2008 & -12.3 & +2.1 & -2.5 \\
\hline & & & BM2012 & -12.3 & +2.1 & -2.5 \\
\hline \multirow[t]{3}{*}{2} & \multirow[t]{3}{*}{ Thickness distribution (Fig. 9) } & \multirow[t]{3}{*}{ Measure } & Hwang & -4.1 & +0.2 & -1.1 \\
\hline & & & BV2008 & -5.3 & -2.3 & -3.3 \\
\hline & & & BM2012 & -7.2 & +11.6 & -1.6 \\
\hline \multirow[t]{3}{*}{3} & \multirow[t]{3}{*}{$\varepsilon_{\theta}$ (Fig. 10) } & \multirow[t]{3}{*}{$\mathrm{FE}$} & Hwang & +1.6 & +2.2 & +1.8 \\
\hline & & & BV2008 & -2.6 & -2.3 & -2.4 \\
\hline & & & BM2012 & -2.6 & -2.3 & -2.4 \\
\hline \multirow[t]{3}{*}{4} & \multirow[t]{3}{*}{$\varepsilon_{z}$ (Fig. 11) } & \multirow[t]{3}{*}{$\mathrm{FE}$} & Hwang & -3100 & +2041 & -238 \\
\hline & & & BV2008 & -656 & +907 & +21 \\
\hline & & & BM2012 & -387 & +649 & +75 \\
\hline \multirow[t]{3}{*}{5} & \multirow[t]{3}{*}{$\varepsilon_{r}$ (Fig. 12) } & \multirow[t]{3}{*}{$\mathrm{FE}$} & Hwang & -31.5 & -10.6 & -18.7 \\
\hline & & & BV2008 & -6.6 & +8.7 & +1.0 \\
\hline & & & BM2012 & +2.5 & +4.0 & +3.0 \\
\hline \multirow[t]{3}{*}{6} & \multirow[t]{3}{*}{$\sigma_{\theta}$ (Fig. 13) } & \multirow[t]{3}{*}{$\mathrm{FE}$} & Hwang & -5.2 & -1.1 & -2.7 \\
\hline & & & BV2008 & -0.7 & +5.6 & +1.5 \\
\hline & & & BM2012 & -0.7 & +5.4 & +1.4 \\
\hline \multirow[t]{3}{*}{7} & \multirow[t]{3}{*}{$\sigma_{z}$ (Fig. 14) } & \multirow[t]{3}{*}{$\mathrm{FE}$} & Hwang & -9.8 & +8.4 & -0.7 \\
\hline & & & BV2008 & +0.5 & +19.9 & +10.3 \\
\hline & & & BM2012 & +0.6 & +19.6 & +10.2 \\
\hline \multirow[t]{3}{*}{8} & \multirow[t]{3}{*}{$t_{p}$ (Fig. 15) } & \multirow[t]{3}{*}{$\mathrm{FE}$} & Hwang & +1.6 & +5.5 & +4.3 \\
\hline & & & BV2008 & -0.8 & +3.4 & -0.4 \\
\hline & & & BM2012 & -1.7 & -0.3 & -0.8 \\
\hline
\end{tabular}

From these comparisons, we can conclude that the three models can be applied for tube material characterization. Nevertheless Hwang's model slightly underestimates the flow stress that would lead to an overestimation of the internal pressure necessary for forming the tube and then to a nonoptimal design of the tube hydroforming process.

\section{Study of the influence of the tube bulging test configuration on the material characterization}

The evolution of the bulge height depends on the test configuration as it is illustrated on Figs. 16 and 17. On the Fig. 16, the die radius is set at $5 \mathrm{~mm}$ and the free length takes the values 40, 50 and $60 \mathrm{~mm}$. On the Fig. 17, the free bulging length is set to $50 \mathrm{~mm}$ and different die radii are considered (5, 7.5 and $10 \mathrm{~mm}$ ). Figures 16 and 17 show clearly that the bulge height increases substantially with a rising of the free length and the die radius. Let note that near the bursting pressure, corresponding to a simulation time close to $0.7 \mathrm{~ms}$, FE results become uncertain leading to very large bulge height far from the reality. An adaptive re-meshing during FE calculation would certainly improve the simulations, but we imposed to work with exactly the same FE model for carrying out the comparisons.
On the Figs. 18, 19, and 20, the hardening curve imposed as input of the FEM is compared with the hardening curve

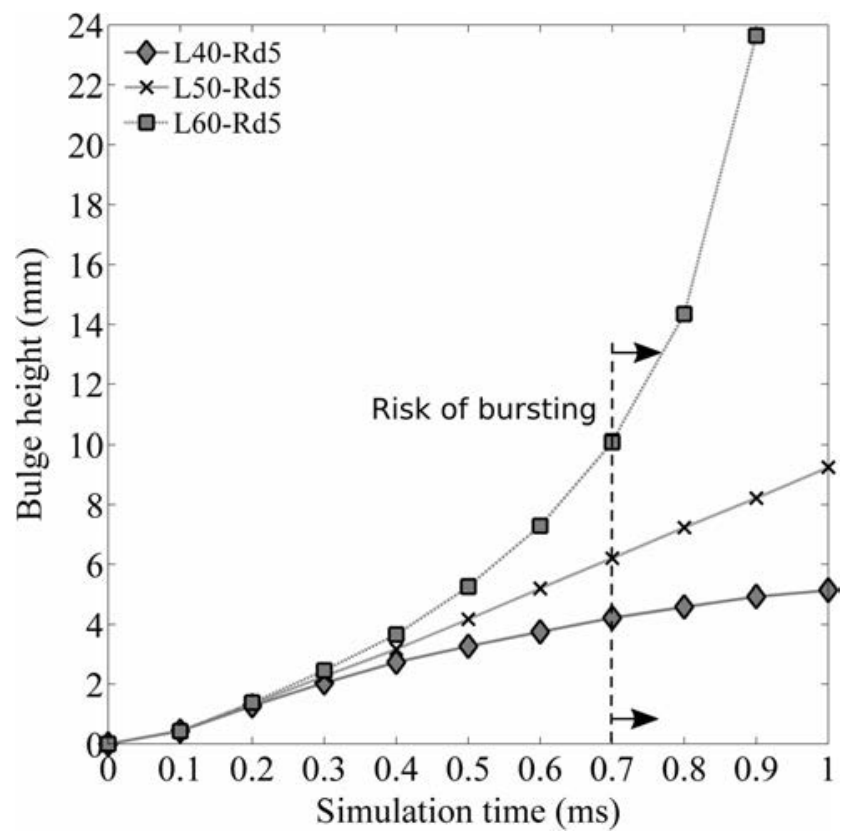

Fig. 16 Bulge height vs. simulation time for the three configurations with Rd. set at $5 \mathrm{~mm}$ 


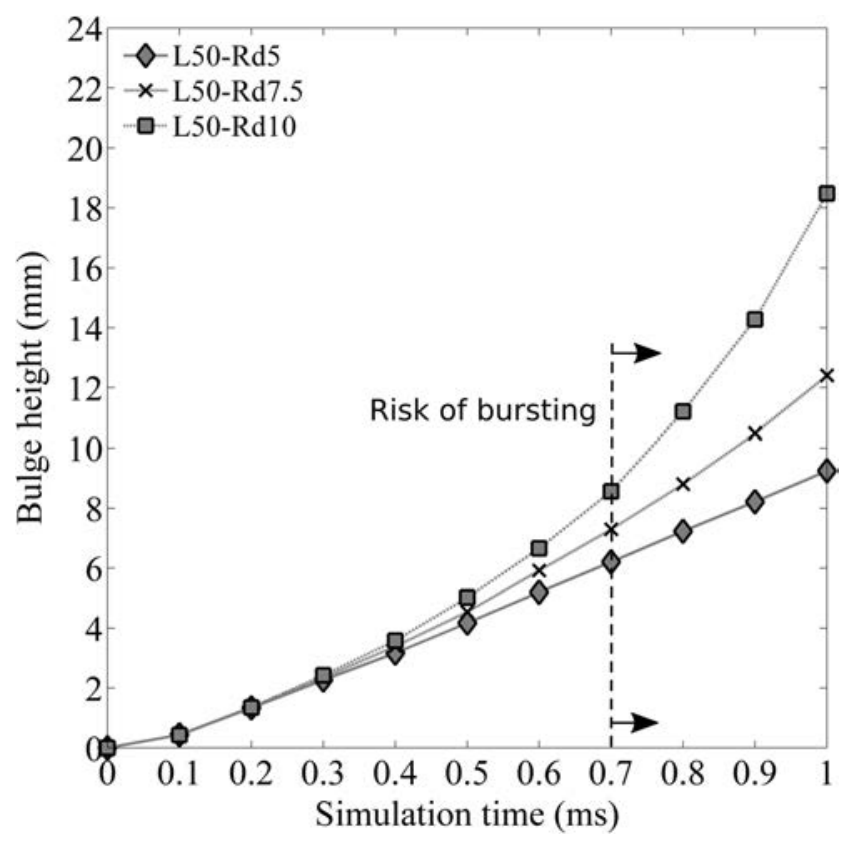

Fig. 17 Bulge height vs. simulation time for the three configurations with $\mathrm{L}$ set at $50 \mathrm{~mm}$

obtained by post processing $(\mathrm{p}, \mathrm{h})$ curves from FE simulations of tube bulging test with the models for different geometrical configurations, following the procedure described in Fig. 7. A quantitative analysis of the results, similar to the one proposed in "Validations and comparisons of models done on the reference configuration of the tube bulging test" section, is given in Table 5 .

By taking into account the die radius, the Hwang's model presents less scattering for the different test configurations (Fig. 18).

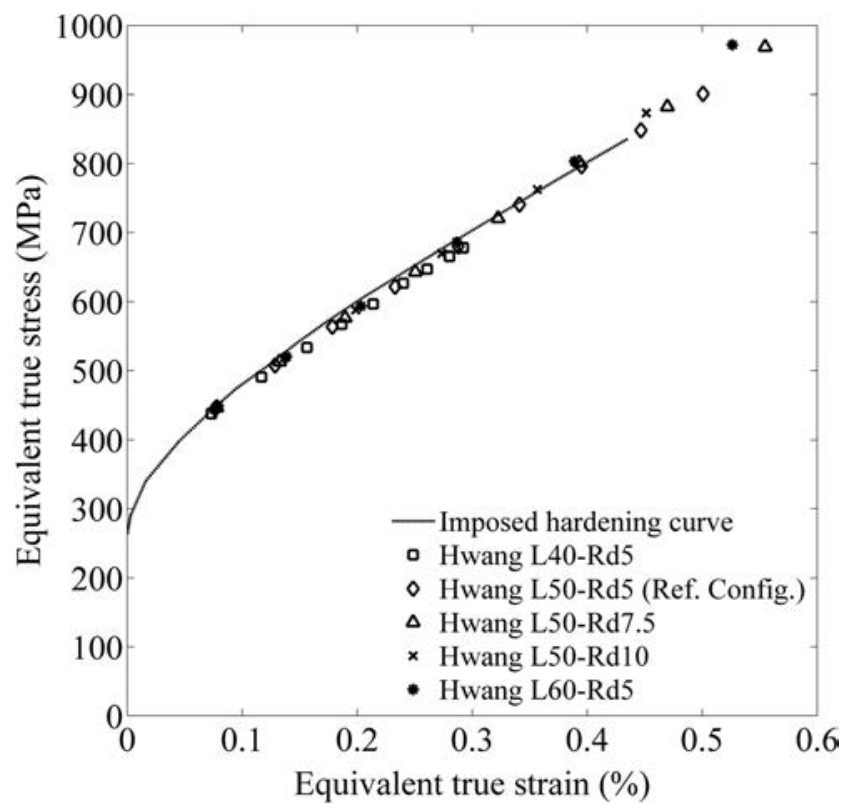

Fig. 18 Hardening curve obtained from the Hwang's model

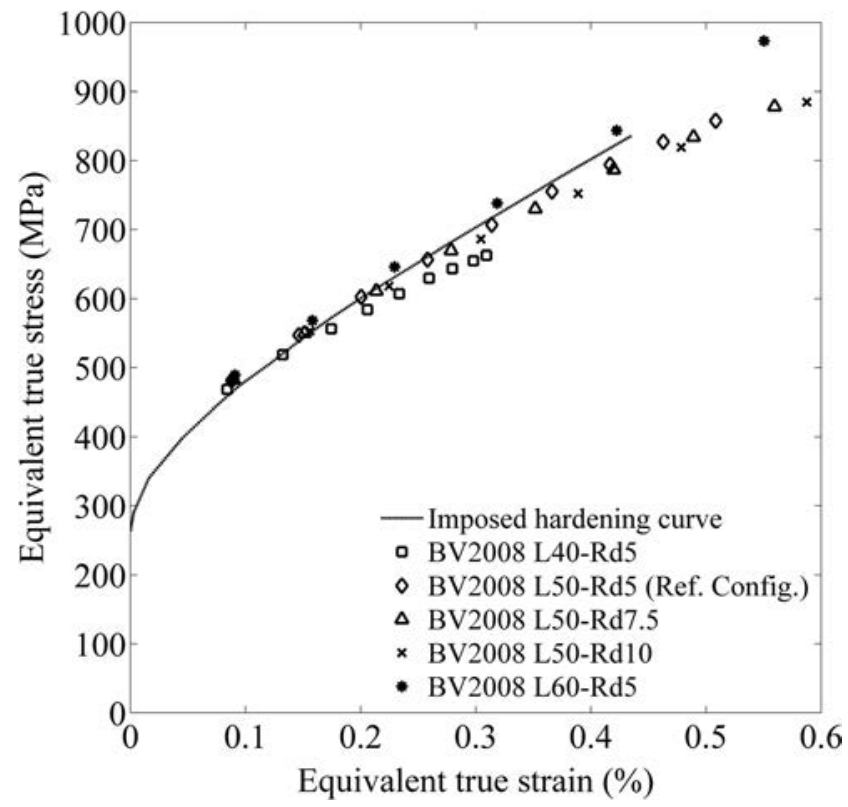

Fig. 19 Hardening curve obtained from the Boudeau \& Velasco's model

The Boudeau-Velasco's and the Boudeau-Malécot's models give very similar and satisfying results (Figs. 19 and 20), even if both of them present more dependence to the test configuration.

The hardening curves obtained with the help of the three theoretical models are very close to the imposed one. Table 5, which reveals deviations less than 5\%, (except in the case of BV2008 and BM2012 models for the configuration with the smallest free bulging length) confirms these observations. A non-negligible influence of the die radius for a small free bulging length explains the large variations observed for

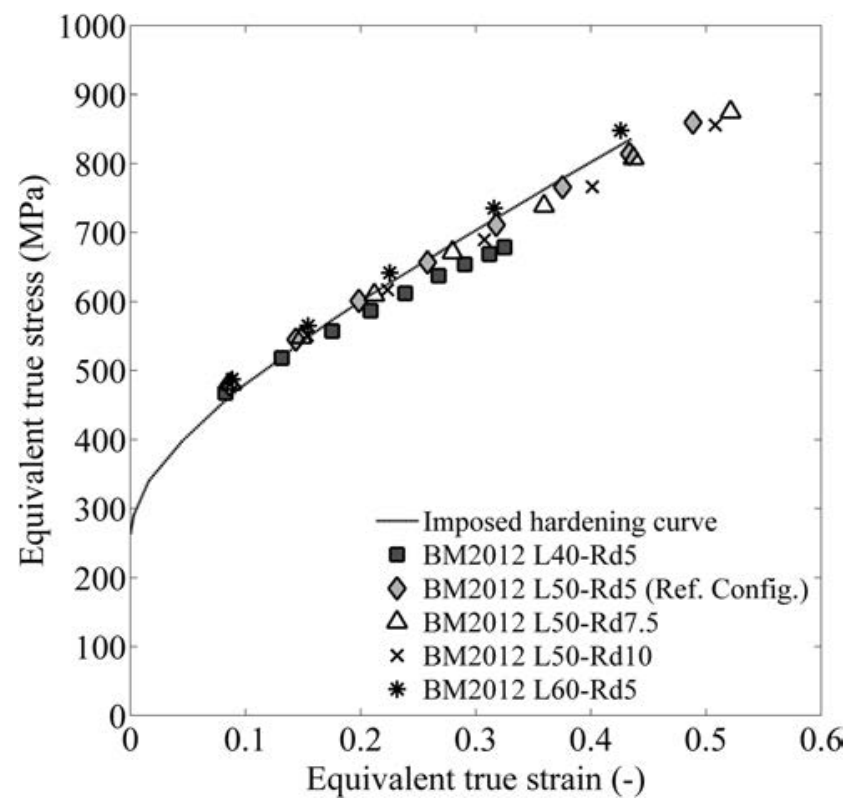

Fig. 20 Hardening curve obtained from the Boudeau \& Malécot's model 
Table 5 Deviations between models and reference for results presented in Figs. 18, 19, and 20

\begin{tabular}{lllllll}
\hline & Reference & L40-Rd5 & L50-Rd5 & L50-Rd7.5 & L50-Rd10 & L60-Rd5 \\
\hline Hwang & Imposed HC & $-2.7 \%$ & $-1.3 \%$ & $-0.7 \%$ & $-0.08 \%$ & $+0.3 \%$ \\
BV2008 & Imposed HC & $-6.6 \%$ & $-1.5 \%$ & $-2.4 \%$ & $-3.1 \%$ & $+2.4 \%$ \\
BM2012 & Imposed HC & $-5.9 \%$ & $-0.9 \%$ & $-1.9 \%$ & $-2.5 \%$ & $+2.4 \%$ \\
\hline
\end{tabular}

models BV2008 and BM2012 for the configuration L40-Rd5. For all other configurations, Table 5 presents deviations less than $5 \%$, which demonstrates the validity of the three studied models.

\section{Application to other tube materials}

In this section the aim is to confirm that the results obtained in the previous part of this work can be generalized to other materials.

\section{Presentation of the studied materials and TBT results}

The three models (Hwang 2007, Boudeau-Velasco 2008 and Boudeau-Malécot 2012) are used for post-processing experimental results obtained from the tube bulging test performed on three different tube materials: Copper, Nickel based alloy and AISI 304. Table 6 gives the tube dimensions and Table 7, the chemical compositions. The pressure - bulge height $(\mathrm{p}, \mathrm{h})$ curves obtained from the tube bulging test are given in Fig. 21 and the resulting stress - strain curves in Fig. 22.

The $(\mathrm{p}, \mathrm{h})$ curve is obtained during the TBT by simultaneously measuring the internal pressure with a pressure sensor (P2VA1/2000Bar from HBM) and the bulge height with a displacement sensor (WA/50 mm from HBM).

Fig. 21 shows that the three tubes present different behaviours. Copper tubes are ductile and present a medium bulge height $(5.5 \mathrm{~mm}$ ) for a reasonable pressure (about $10 \mathrm{MPa}$ ). Stainless steel tubes present an important bulge height (about $9 \mathrm{~mm}$ ) but need a higher pressure (about $30 \mathrm{MPa}$ ). The Nickel based alloy is the less ductile and is difficult to deform: a bulge height of only $1.5 \mathrm{~mm}$ is obtained for a pressure of nearly $50 \mathrm{MPa}$.

Table 6 Tubes dimensions

\begin{tabular}{llll}
\hline Material & $\begin{array}{l}\text { External diameter } \\
(\mathrm{mm})\end{array}$ & $\begin{array}{l}\text { Initial thickness } \\
(\mathrm{mm})\end{array}$ & Observation \\
\hline Copper & 35 & 0.9 & Extruded \\
AISI304 & 35 & 1.0 & Rolled-welded \\
INCONEL & 38.1 & 1.0 & Rolled-welded \\
\hline
\end{tabular}

\section{Post-processing of TBT measures considering isotropic materials}

The three models used for post-processing the tube bulging test results give very similar stress - strain curves. For ductility, AISI 304 material is the best with a true strain at rupture of about 0.5 ; for Copper, it is approximately 0.3 and only 0.1 for the INCONEL. AISI 304 is the harder material with a true stress at rupture of about $1100 \mathrm{MPa}$; for the Nickel based alloy, it is about $900 \mathrm{MPa}$ and only $300 \mathrm{MPa}$ for the Copper. The following yield stresses are identified: $100 \mathrm{MPa}$ for Copper, $300 \mathrm{MPa}$ for AISI 304 and $600 \mathrm{MPa}$ for INCONEL. In conclusion, the three models permit to get the hardening curve for a wide variety of materials.

\section{Taking into account initial anisotropy of material}

Anisotropy was not our main objective. The reasons were motivated by the following facts:

1) $316 \mathrm{~L}$ used in the main part of this study is nearly isotropic considering its Lankford coefficients: $r_{0}=1.01$; $r_{45}=0.91 ; r_{90}=0.91$ [23]. Moreover, $316 \mathrm{~L}$ is most of the time considered as isotropic in the literature [24].

2) The theoretical models used in this work are based on geometrical consideration for the calculation of the strain tensor components, and on elementary volumes equilibrium for the evaluation of the stress tensor components, without any concern of isotropy or anisotropy.

The studied theoretical models permit the evaluation of the components of the true strain and true stress tensors. Thus, it is possible to decide, in another step, to take into account or not anisotropy for the calculation of the equivalent true stress and the equivalent true strain.

In case of anisotropic material, more data are required, like the Lankford coefficients for example. Another requirement is the choice of a plastic criterion for the calculation of the equivalent stress from the components of the stress tensor.

If the material is isotropic, the equivalent true stress is calculated with the von Mises plastic criterion whose expression is:

$\bar{\sigma}=\sqrt{\sigma_{\theta}^{2}+\sigma_{z}^{2}-\sigma_{\theta} \sigma_{z}}$ 
Table 7 Chemical compositions of the tube materials

\begin{tabular}{|c|c|c|c|c|c|c|c|c|c|c|}
\hline $\mathrm{W} \%$ & $\mathrm{Cu}$ & $\mathrm{P}$ & & $\mathrm{Ni}$ & $\mathrm{Cr}$ & Mo & & $\mathrm{Fe}$ & $\mathrm{Cr}$ & $\mathrm{Ni}$ \\
\hline Copper & 99.9 & 0.03 & Nickel alloy & 65 & 22 & 9 & Stainless Steel & 70 & 19 & 9 \\
\hline
\end{tabular}

If the material is anisotropic, one has to choose an anisotropic plastic criterion. Amongst the numerous existing plastic criteria, the Hill 1948 and the Hosford 1979 criteria are considered [25].

Hill 1948 criterion presents the following expression:

$\bar{\sigma}=\sqrt{\sigma_{\theta}^{2}+\frac{r_{0}\left(1+r_{90}\right)}{r_{90}\left(1+r_{0}\right)} \sigma_{z}^{2}-\frac{2 r_{0}}{1+r_{0}} \sigma_{\theta} \sigma_{z}}$

The expression for the Hosford 1979 plastic criterion is:

$\bar{\sigma}=\left[\frac{1}{1+r_{0}}\left|\sigma_{\theta}\right|^{a}+\frac{r_{0}}{r_{90}\left(1+r_{0}\right)}\left|\sigma_{z}\right|^{a}+\frac{r_{0}}{1+r_{0}}\left|\sigma_{\theta}-\sigma_{z}\right|^{a}\right]^{1 / a}$

where exponent $a$ equals 6 for FCC materials and 8 for $\mathrm{BCC}$ materials.

The corresponding equivalent true strain is obtained by considering the constancy of the plastic work, which is expressed below:

$\bar{\sigma} \cdot \bar{\varepsilon}=\underline{\sigma}: \underline{\varepsilon}=\sigma_{\theta} \varepsilon_{\theta}+\sigma_{z} \varepsilon_{z}$

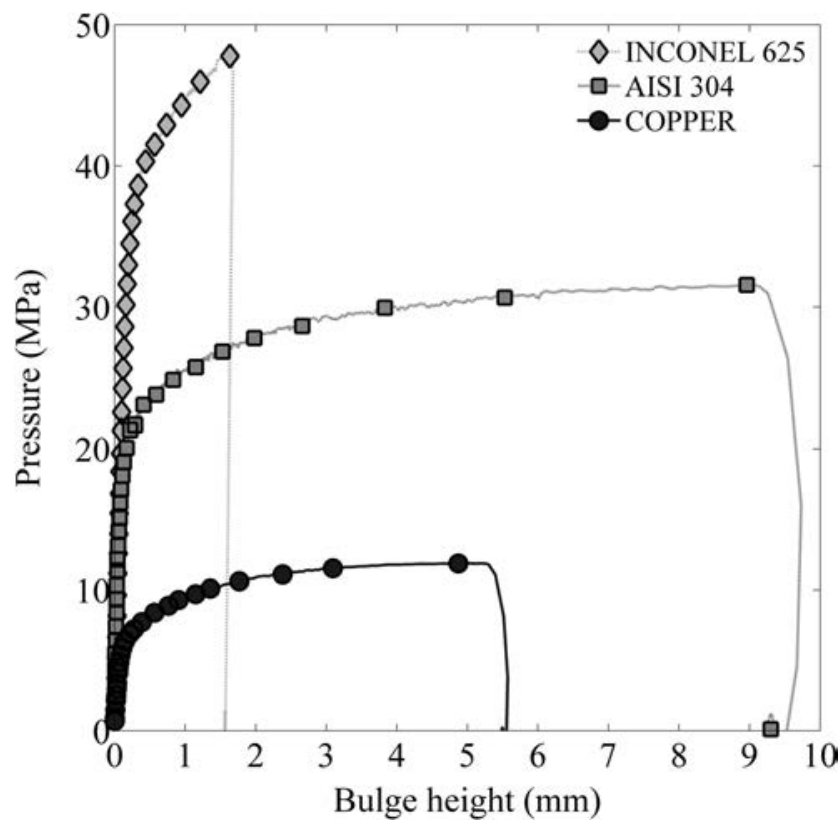

Fig. 21 Pressure - Bulge Height curves measured during the tube bulging test performed on tubes made of INCONEL625, AISI304 and Copper
For the illustration, the INCONEL, a FCC material, is chosen as it presents the largest anisotropy of the three studied materials with the following Lankford coefficients: $r_{0}=0.86$; $r_{45}=1.27 ; r_{90}=1.0$. Figure 23 presents the resulting hardening curves.

Therefore, three elements can be observed:

1) The three models permit to take into account the anisotropy and to obtain a hardening curve depending on the chosen plastic criterion.

2) The different hardening curves are very close to one another. The anisotropy of INCONEL is revealed by the $r_{45}$ Lankford coefficient, which is not present in the expression of Hill 1948 and Hosford 1979 criteria.

3) The hardening curves calculated with von Mises and Hill 1948 criteria are nearly overlaid as $r_{0}$ and $r_{90}$ are close to 1 .

With additional data, it would be possible to consider advanced plastic criteria, such as Barlat family plastic criteria [25].

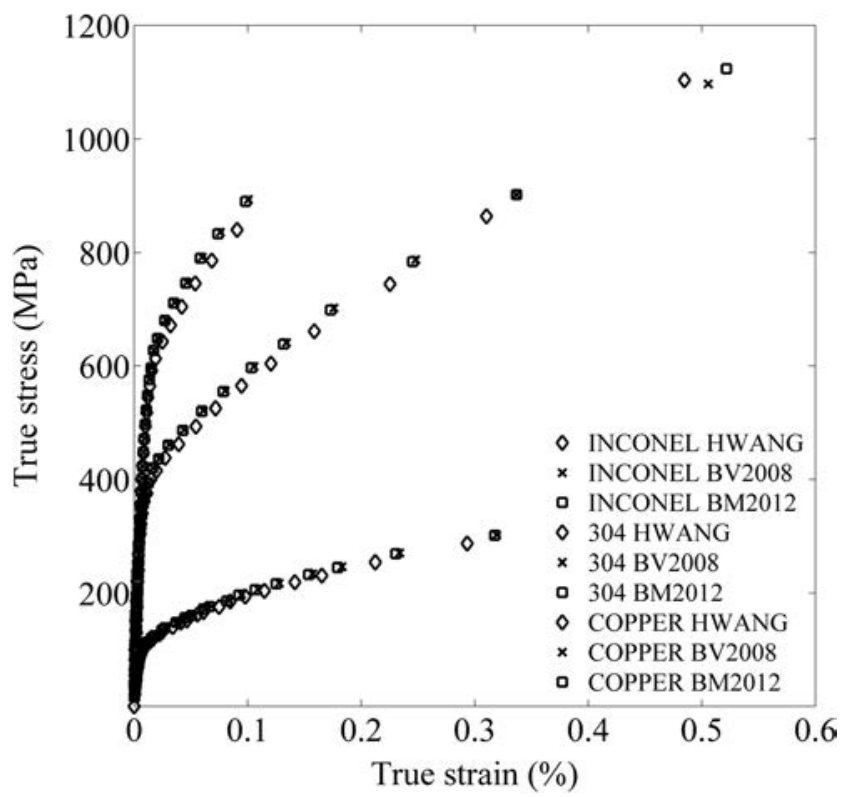

Fig. 22 Resulting stress - strain curves for tubes made of Copper, INCONEL625 and AISI304 tested with the tube bulging test. The experimental results of Fig. 21 have been post-processed with the Hwang 2007, Boudeau-Velasco 2008 and Boudeau-Malécot 2012 models 
Fig. 23 Resulting stress - strain curves for tubes made of INCONEL625 tested with the tube bulging test. The experimental results of Fig. 21 have been post-processed with the Hwang 2007, Boudeau-Velasco 2008 and Boudeau-Malécot 2012 models using Von Mises or Hill48 criteria
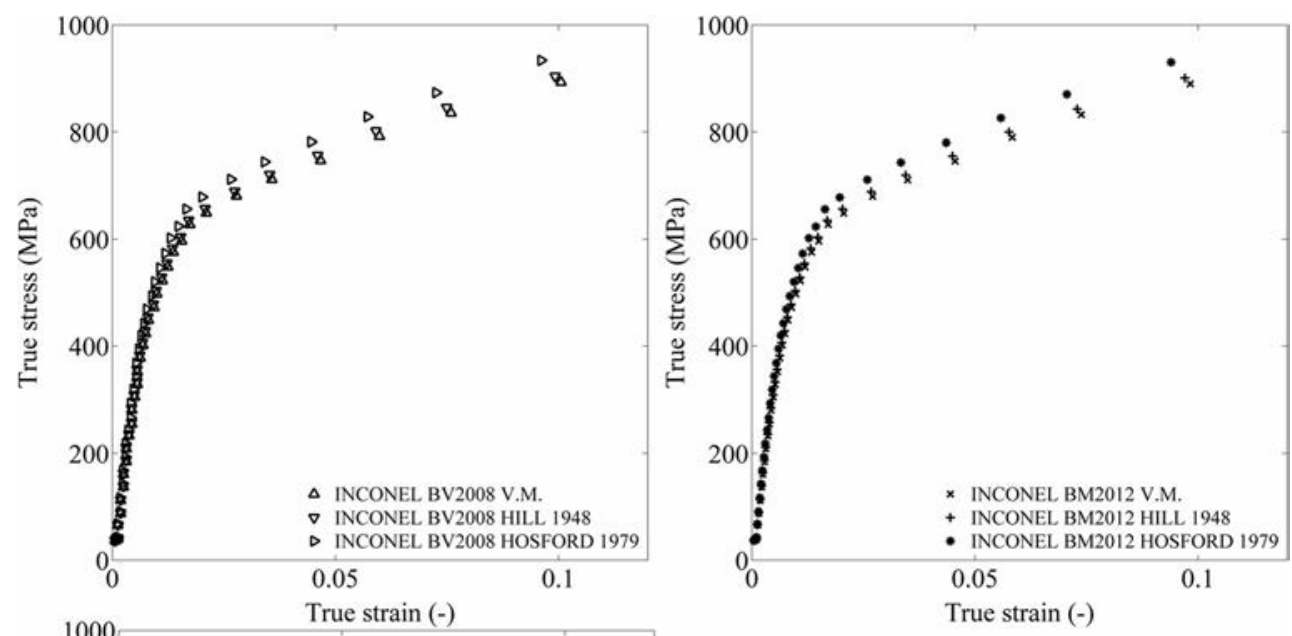

\section{Conclusions}

In this paper, three models of the literature for post-processing experimental results obtained from the tube bulging test are studied: Hwang's model, Boudeau-Velasco's model and Boudeau-Malécot's model.

Their programming is validated based on postprocessing pressure - bulge height data obtained by FE simulations of the tube bulging test. Their validity for the strain-stress curve characterization of a tube material expanded with an internal pressure is demonstrated. Local comparisons in terms of strain and stress components are carried out. Surprisingly the simplest model (Boudeau-Malécot's model) gives very good results:

- Deviations less to 5\% for the evaluation of the radial and circumferential true stain

- Deviations not more than $10 \%$ for the meridian and circumferential true stresses

- A large deviation for the meridian true strain but very less than with Hwang2007 model
As specified in the introduction, no rules exist for performing such tube material characterization. Therefore, different configurations are tested based on finite element simulations. It appears that the Hwang's model is less influenced by the test configuration, since it takes into account the die radius in the geometrical modelling. And it gives very good results for the stress-strain curve, even if the evaluation of the meridian strain is not realistic. The other two models studied in the present work present more dependence to the tube bulging test configuration, but give satisfying results anyway, with deviations less than 5\% when the free bulging length/die radius ratio is sufficiently important (more than 8 ).

The Boudeau-Malécot's model is the best compromise in terms of simplicity and efficiency for tube material characterization. Indeed, this model treats experimental measures with a simple spreadsheet application, that present a great advantage in an industrial context.

The three models are used for post processing the experimental measures obtained from TBT carried out on tubes made of copper, stainless steel and Nickel based alloy. Their capability to get the hardening curve is demonstrated. The three models give very close hardening curve so the 
Boudeau-Malécot model, which permits to post process experimental results with a spreadsheet application, is an interesting approach for an industrial use. Moreover, their results can be used with different plastic criteria since anisotropy data are available.

Acknowledgements The authors would like to thank J. Kress for the realized works during his internship within the APERAM Company.

Funding This work was supported by the Ministry of Higher Education and Research, the Europe and the Franche-Comte region (FEDER-Project "Plateform R\&D de fabrication et de caractérisation de composants et $\mu$-composants pour les transports terrestres").

\section{Compliance with ethical standards}

Declaration of conflicting interests The authors declare that there is no conflict of interest.

\section{References}

1. Lang LH, Wang ZR, Kang DC et al (2004) Hydroforming highlights: sheet hydroforming and tube hydroforming. J Mater Process Tech. 151(1-3):165-177

2. Chu E, Xu Y (2004) Hydroforming of aluminum extrusion tubes for automotive applications. Part II: process window diagram. Int J Mech Sci 46(2):285-297

3. Mirzaali M, Seyedkashi SMH, Liaghat GH et al (2012) Application of simulated annealing method to pressure and force loading optimization in tube hydroforming process. Int J Mech Sci 55(1):78-84

4. Carleer B, van der Kevie G, de Winter L et al (2000) Analysis of the effect of material properties on the hydroforming process of tubes. $\mathrm{J}$ Mater Process Tech. 104(1):158-166

5. Song WJ, Heo SC, Ku TW et al (2010) Evaluation of effect of flow stress characteristics of tubular material on forming limit in tube hydroforming process. Int J Mach Tool Manu 50(9):753-764

6. Suttner S, Merklein M (2016) Experimental and numerical investigation of a strain rate controlled hydraulic bulge test of sheet metal. J Mater Process Tech. 235:121-133

7. Kress J (2013) Caractérisation mécanique des tubes. Dissertation, ENSMM

8. Dufour JE, Leclerq S et al (2016) 3D surface measurement with isogeometric stereocorrelation - application to complex shapes. Opt Lasers Eng 87:146-155
9. Khalfallah A, Oliveira MC, Alves JL et al (2015) Mechanical characterization and constitutive parameter identification of anisotropic tubular materials for hydroforming applications. Int J Mech Sc 104: 91-103

10. Sokolowski T, Gerke K, Ahmetoglu M et al (2000) Evaluation of tube formability and material characteristics: hydraulic bulge testing of tubes. J Mater Process Tech. 98(1):34 40

11. Fuchizawa S, Narazaki M (1993) Bulge test for determining stressstrain characteristics of thin tubes. In: Proc Fourth Int Conf Tech Plast Adv Tech Plast, no 1, pp 488-93

12. Bortot P, Ceretti E, Giardini C (2008) The determination of flow stress of tubular material for hydroforming applications. J Mater Process Tech. 203(1-3):381-388

13. Hwang YM, Lin YK, Altan T (2007) Evaluation of tubular materials by a hydraulic bulge test. Int J Mach Tool Manu 47(2):343-351

14. Lianfa Y, Cheng G (2006) A simple experimental tooling with internal pressure source used for evaluation of material formability in tube hydroforming. J Mater Process Tech 180(1-3):310-317

15. Velasco R, Boudeau N (2008) Tube bulging test: theoretical analysis and numerical validation. J Mater Process Tech 205(1-3):51-59

16. Koç M, Altan T et al (2001) On the characteristics of tubular materials for hydroforming - experimentation and analysis. Int J Mach Tool Manu 41(5):761-772

17. He Z, Yuan S, Lin Y et al (2014) Analytical model for tube hydrobulging test, part I: models for stress components and bulging zone profile. Int J Mech Sci 87:297-306

18. Kuwabara T (2005) Anisotropic plastic deformation of extruded aluminum alloy tube under axial forces and internal pressure. Int $\mathrm{J}$ Plasticity 21(1):101-117

19. Strano M, Altan T (2004) An inverse energy approach to determine the flow stress of tubular materials for hydroforming applications. J Mater Process Tech 146(1):92-96

20. Hwang YM, Wang CW (2009) Flow stress evaluation of zinc copper and carbon steel tubes by hydraulic bulge tests considering their anisotropy. J Mater Process Tech 209(9):4423-4428

21. Boudeau N, Malécot P (2012) A simplified analytical model for post-processing experimental results from tube bulging test: theory, experimentations, simulations. Int J Mech Sci 65(1):1-11

22. Hwang YM, Lin YK (2002) Analysis and finite element simulation of the tube bulge hydroforming process. J Mater Process Tech 125 : $821-825$

23. Talyan V, Wagoner RH, Lee JK (1998) Formability of stainless steel. Metal Mater Trans A 29A:2161-2171

24. Lacombe P, Baroux B, Beranger G (1993) Stainless steels, les Ulis : Les éditions de physique, $\mathrm{p} 714$

25. Banabic D, Bunge HJ, Pöhlandt K, Tekkaya AE (2000) Formability of metallic materials. Springer, Berlin 